\title{
Diagnosis of intravascular catheter infection Philippe Eggimann
}

\begin{abstract}
Purpose of review
To review the distinction between catheter-related and catheter-associated infections and to report the recent advances in the methods used for their diagnosis.

Recent findings

The distinction between device-associated and devicerelated infections affects the effective benchmarking of the rates of both types of infection. Numerous microbiological methods have been described to diagnose these infections. Studies comparing the performance of microbiological methods that avoid the removal of the intravascular device have recently suggested that they may be effective in daily life.

\section{Summary}

The present review summarizes recent advances in the methods currently available to diagnose intravascular catheter-related infections and their performance at the bedside.

\section{Keywords}

catheter, catheter-related infection, diagnosis, nosocomial infection
\end{abstract}

Curr Opin Infect Dis 20:353-359. (c) 2007 Lippincott Williams \& Wilkins.

Department of Adult Intensive Care Medicine and Burn Centre, Centre Hospitalier Universitaire Vaudois (CHUV), Lausanne, Switzerland

Correspondence to Philippe Eggimann, MD, Department of Adult Intensive Care Medicine and Burn Centre, Centre Hospitalier Universitaire Vaudois (CHUV), BH 08-609, Bugnon 46, CH-1011 Lausanne, Switzerland

Tel: +41 21314 2923; fax: +4121314 3045;

e-mail: philippe.eggimann@chuv.ch

Current Opinion in Infectious Diseases 2007, 20:353-359

\section{Abbreviations}

CVC central venous catheter

ICU intensive care unit

(C) 2007 Lippincott Williams \& Wilkins 0951-7375

\section{Introduction}

Infections associated with the use of intravascular catheters or devices represent $10-20 \%$ of all nosocomial infections. They may complicate the stay of up to $10 \%$ of intensive care unit (ICU) patients. Almost all patients staying in an ICU require at least one intravascular device for fluid/drug administration, and approximately half are central venous catheters (CVCs) [1]. According to data from the National Nosocomial Infections Surveillance system, it is estimated that at least 48600 ICU patients develop a CVC-related bloodstream infection every year in US ICUs (approximately five episodes per 1000 catheter-days). These infections, mostly caused by coagulase-negative staphylococci, Staphylococcus aureus, Enterococcus species, and Candida species, are associated with considerable morbidity (prolonged length of stay and increased costs) and mortality $\left[2^{\circ}\right]$. Although debated by experts with regard to magnitude, the attributable mortality of these infections may correspond to $5000-15000$ deaths directly caused by catheter-related infections; the benchmarking of rates is currently included in the assessment of quality of care in many institutions $\left[3^{\circ}\right]$.

The diagnosis of infections attributable to the use of intravascular catheters or devices is the subject of intense clinical research. There is, however, no consensus on a true gold standard, and the accuracy of numerous microbiological methods has generated vigorous debate among experts [4]. In addition, the variability in the definitions used over the past decades has not simplified the understanding of the literature $\left[5^{\circ}\right]$.

In this context, the distinction between device-associated and device-related infections proposed in the 2002 guidelines for the prevention of intravascular catheter-related infections provided a useful tool [6]. Infection rates vary according to the type of surveillance. In studies designed to study complications associated with the use of intravascular devices, epidemiological definitions frequently result in higher infection rates. In studies dedicated to device surveillance, systematic microbiological investigation allows the determination of infection rates directly related to the colonization or infection of the device [7].

\section{Diagnosis of infections associated with or related to vascular access}

Before reviewing the methods available to diagnose intravascular catheter-related infections, it is important 
to summarize the different definitions commonly used in the literature.

\section{Definitions for vascular access-associated and related infections}

Infections linked to the use of intravascular devices include exit-site infections, catheter colonization and both catheter-associated and catheter-related infections [6,8-15] (Table 1).

Catheter-associated infections include primary bloodstream infections and clinical sepsis, which are epidemiologically associated with the use of intravascular devices [6,7]. It should be emphasized that in the absence of device culture, defervescence after the removal of an implicated catheter from a patient with primary bloodstream infection is considered indirect evidence of catheter-associated bloodstream infection. Comparisons between infection rates in different types of ICUs are more accurate when infections are reported as incidence densities associated with the use of intravascular devices. According to this method, widely diffused by the National Nosocomial Infections Surveillance system and using epidemiological definitions, catheter-associated infections range between 2.3 and 16.8 episodes per 1000 CVC-days [1]. This may overestimate the rate of infections related to intravascular devices, but is probably more representative of daily life. This method allows the benchmarking of rates of infection after eventual adjustment for the case mix without the need for sophisticated laboratory work-up. Although included in some reports, secondary bacteraemia, which

Table 1 Definitions of infections potentially related to vascular access ${ }^{a}$

\begin{tabular}{|c|c|}
\hline Type of infection & Criteria \\
\hline Exit-site infection & $\begin{array}{l}\text { Clinically documented: a clinical infection (erythema, tenderness, induration or purulent } \\
\text { discharge) at the skin insertion site } \\
\text { Microbiologically documented: a positive (semi) quantitative catheter culture in the presence } \\
\text { of clinical signs of infection at the insertion site }\end{array}$ \\
\hline Catheter colonization & $\begin{array}{l}\text { In the absence of clinical signs of infection at the insertion site, growth of microorganisms } \\
\text { on the device according to microbiological criteria from quantitative (technique [8], } \\
\text { sonication [9], vortexing technique [10]) or semi-quantitative (roll-plate technique [11]) } \\
\text { cultures. }\end{array}$ \\
\hline Positive blood culture & Microorganism potentially pathogen cultured from one or more blood cultures ${ }^{b}$ \\
\hline Bloodstream infection & $\begin{array}{l}\text { Positive blood culture with at least one of the following clinical signs or symptoms: } \\
\text { Fever }\left(>100.4^{\circ} \mathrm{F} ;>38^{\circ} \mathrm{C}\right) \text { or hypothermia }\left(<98.6^{\circ} \mathrm{F} ;<37^{\circ} \mathrm{C}\right) \\
\text { Chills } \\
\text { Low blood pressure (systolic blood pressure } \leq 90 \mathrm{mmHg} \text { or decrease }>40 \mathrm{mmHg} \text { from baseline) } \\
\text { In the absence of catheter culture, defervescence after removal of an implicated catheter } \\
\text { from a patient with primary bloodstream infection is considered as indirect evidence } \\
\text { of catheter-associated bloodstream infection }\end{array}$ \\
\hline Primary bloodstream infection & $\begin{array}{l}\text { Laboratory-confirmed bloodstream infection or clinical sepsis occurring without documented } \\
\text { distal source of infection }\end{array}$ \\
\hline Secondary bloodstream infection & $\begin{array}{l}\text { Laboratory-confirmed bloodstream infection occurring in the presence of another documented } \\
\text { infection }\end{array}$ \\
\hline Clinical sepsis & $\begin{array}{l}\text { Requires one of the following signs with no other recognized cause: } \\
\text { Fever }\left(>100.4^{\circ} \mathrm{F} ;>38^{\circ} \mathrm{C}\right) \text { or hypothermia }\left(<98.6^{\circ} \mathrm{F} ;<37^{\circ} \mathrm{C}\right) \\
\text { Low blood pressure (systolic blood pressure } \leq 90 \mathrm{mmHg} \text { or decrease }>40 \mathrm{mmHg} \\
\text { from baseline) } \\
\text { Oliguria }(<20 \mathrm{ml} / \mathrm{h}) \\
\text { and the presence of all of the following conditions: } \\
\text { Blood cultures not performed or no organism detected in blood } \\
\text { No apparent infection at another body site } \\
\text { Physician institutes therapy for sepsis }\end{array}$ \\
\hline Catheter-associated bloodstream infection & Primary bloodstream infection or clinical sepsis in the presence of an intravascular device \\
\hline Catheter-related bloodstream infection & $\begin{array}{l}\text { Laboratory-confirmed bloodstream infection in a patient with an intravascular access } \\
\text { with at least one positive blood culture obtained from a peripheral vein, with clinical } \\
\text { manifestations of infection (fever, chills or hypotension) and no apparent source of the } \\
\text { bloodstream infection except the vascular access, and with one of the microbiological } \\
\text { methods described in Table } 2 \text { : } \\
\text { A positive semi-quantitative culture }(>15 \text { cfu/catheter segment) with the same organism [11] } \\
\text { A positive quantitative culture }\left(>10^{3} \mathrm{cfu} / \text { catheter segment) with the same organism [8-10] }\right. \\
\text { Paired quantitative blood cultures with a } \geq 5: 1 \text { ratio device versus peripheral [12] } \\
\text { Differential period of device culture versus peripheral blood culture positivity of }>2 \mathrm{~h} \text { [13] }\end{array}$ \\
\hline
\end{tabular}

cfu, Colony-forming units.

${ }^{a}$ Adapted from $[6,12,14,15]$.

b One of the following:

Common skin contaminant (diphtheroids, Bacillus spp., Propionibacterium spp., coagulase-negative staphylococci, or micrococci) cultured from two or more blood cultures drawn on separate sets.

Common skin contaminant (diphtheroids, Bacillus spp., Propionibacterium spp., coagulase-negative staphylococci, or micrococci) cultured from one or more blood culture from a patient with a vascular access, and the physician institutes appropriate antimicrobial therapy.

Positive antigen test on blood and signs and symptoms with positive laboratory results are not related to an infection at another site. 
is related to another documented focus of infection, should not be considered as being catheter related.

Catheter-related infections include colonization of the device by microorganisms, exit-site infection and microbiologically confirmed device-related bloodstream infection. In the absence of a gold standard reference technique, microbiological criteria are the subject of intense clinical research, and their clinical relevance is currently widely discussed among experts [4]. Maki et al. $\left[16^{\circ \bullet}\right.$ ] recently extracted the risk of bloodstream infections associated with different intravascular devices from a systematic review of 200 published prospective studies. Using microbiologically based criteria, they showed that all types of intravascular devices are at risk of devicerelated bloodstream infections. As rates of infections are likely to be used for benchmarking, they showed that expressing the risks of device-related bloodstream infections per 1000 device-days allows for more meaningful estimates of risk than measuring bloodstream infections per 100 devices. Peripheral and midline intravenous catheters are associated with the lowest rates of infection $(0.1 \%, 0,4 \%$ and $0.5,0.2$ per 1000 device-days, respectively). The rates are slightly higher for arterial catheters used for haemodynamic monitoring $(0.8 \%, 1.7$ per 1000 device-days) and peripherally inserted CVCs in hospitalized patients $(2.4 \%, 12.1$ per 1000 device-days). According to these data, the rates are higher for nonimpregnated GVCs inserted in critically ill patients (4.4\%, 2.7 per 1000 device-days). The highest rates are reported for short-term non-cuffed and non-tunneled haemodialysis catheters $(8.0 \%, 4.8$ per 1000 devicedays), for intra-aortic balloon pumps $(3.0 \%, 7.3$ per 1000 device-days), and for left ventricular assist devices (21.6\%, 2.1 per 1000 device-days).

\section{Clinical diagnosis of infections associated with vascular access}

Except for some exit-site infections, the clinical diagnosis of infections related to vascular access is difficult. Most clinical signs are insensitive, non-specific or late, such as septic thrombophlebitis, endocarditis or septic emboli. Accordingly, they are clinically suspected when clinical sepsis develops without other obvious sources of infection.

The concept of 'clinical sepsis' is included in the surveillance definitions proposed by the Centers for Disease Control and Prevention for primary bloodstream infections to take into account sepsis episodes in which no pathogen has been cultured from blood [6]. This entity that is used for epidemiological purposes is relatively close to the definition of the syndromes of systematic inflammatory response, severe sepsis and septic shock in response to an inflammatory or infectious process $[17,18]$.
The definition is sensitive but non-specific. The impact of clinical sepsis is very close to that of a microbiologically documented episode. In a prospective surveillance study of nosocomial infections in 1068 patients who stayed in a medical ICU for more than $48 \mathrm{~h}$, Hugonnet et al. [7] analysed 109 episodes of bloodstream infections, including 32 episodes of microbiologically documented catheter-related infections and 77 of clinical sepsis. Exposure to central lines and arterial lines, censored at the time of the first episode of bloodstream infection, was similar in patients with a microbiologically documented episode and those with clinical sepsis, but was significantly lower in patients without bloodstream infection. The median ICU length of stay was longer in patients with microbiologically documented bloodstream infections (15.5 days; range 4-67) and clinical sepsis (14.0 days; range $3-48$ ) than among patients without bloodstream infection (4 days; range 2-134; both $P<0.001)$. The hospital mortality rates in patients without a bloodstream infection, with a microbiologically confirmed bloodstream infection, and with clinical sepsis were $22.7,32.1$, and $39.7 \%$, respectively $(P=0.01)$. These data strongly suggest that clinical sepsis and primary bloodstream infection microbiologically related to intravascular devices have the same impact.

If confirmed by large multicentre clinical trials, these data may justify the aggressive strategy currently applied at the bedside in many ICUs, where suspect intravascular devices are removed or exchanged over a guidewire in all cases of clinical sepsis associated with severe sepsis or septic shock developing without another obvious source of infection. This technique may increase the likelihood of infection of the new catheter, but reduces the rate of complications associated with CVC insertion in a new site [19]. Removal of the exchanged device with further insertion at a new site is then only required in the presence of a positive culture of the exchanged device [20].

Only approximately a quarter to one third of these episodes will be demonstrated to be caused by a microbiologically documented infection of the intravascular device, and experts suggested that '... nontunneled GVCs should not be routinely removed in patients with mild to moderate disease' [21,22].

Rijinders et al. [23] studied the impact of a clinical algorithm designed to avoid catheter removal in ICU patients developing clinical sepsis. Of 140 patients potentially eligible, $80(55 \%)$ were excluded for haemodynamic instability, confirmed bacteraemia or local signs of infection at the insertion site. During the 10 days after inclusion, only 16 CVCs (38\%) were removed in the 'watchful waiting' arm (32 patients) compared with 38 $(100 \%)$ in the control group (32 patients), $P<0.01$. 
A catheter-related bloodstream infection developed in three $(1 \%)$ compared with two $(1 \%)$ patients, respectively, but in 47 (25\%) of those excluded before randomization. This preliminary result confirms that some CVCs may be maintained, and further studies should now confirm the usefulness of this approach.

In this context, microbiological techniques likely to provide early laboratory confirmation of the clinical suspicion of device-related infection should be improved to avoid unnecessary intravascular device removal or exchange.

\section{Microbiological diagnosis of infections related to vascular access}

A large majority of primary bloodstream infections originates from infected vascular access, but a microbiological confirmation of an infection of the device is required to be scored as an intravascular access-related bloodstream infection.

\section{Microbiological methods}

Many microbiological methods have been described to diagnose intravascular access-related infections, but in the absence of a true gold standard there is currently no consensus of opinion on which method to use. These methods may be divided schematically into those requiring study of the catheter itself and those that avoid removing the device. They are the subject of intense clinical research, and meta-analyses on the performance of some of these methods have recently appeared in the literature [8-13,15,21,24-35] (Table 2).

Of particular interest is the fact that paired qualitative blood cultures drawn from the device and venipunctures and cultures of swabs obtained from the skin insertion site or from the hub, which are less sophisticated from a microbiological point of view but are also cheaper, are characterized by a high specificity and have the highest negative predictive value. This may explain partly why more sophisticated microbiological methods with high sensitivity and the highest positive predictive value and accuracy are currently not widely used.

\section{Comparison of methods}

Some microbiological techniques have been carefully compared in a few prospective studies.

In a prospective cohort study on 128 CVCs suspected of causing catheter-related bloodstream infection, Kite $\mathrm{et} \mathrm{al}$. [30] compared the performance of four methods that allowed the device to remain in situ. The sensitivity of the Gram stain and acridine-orange leukocyte cytospin test was $96 \%$ and the specificity was $92 \%$. By comparison, the tip-roll, tip-flush, and endoluminal-brush methods had sensitivities of 90,95 , and $92 \%$, respectively, with specificities of 55,76 , and $98 \%$, respectively. From these data, the authors concluded that the Gram stain and acridine-orange leukocyte cytospin test are simple and rapid methods for the diagnosis of catheter-related bloodstream infection, which compare favourably with other methods.

In a prospective cohort study of 125 CVCs suspected of causing catheter-related bloodstream infection, Catton et al. [36] compared the performance of three methods that allowed the device to remain in situ. The sensitivities of the endoluminal brush, of quantitative culture blood cultures, and of the differential time to positivity were 100,89 , and $72 \%$, respectively, with corresponding specificities of 89,97 , and $95 \%$, respectively. Blood could be directly aspirated from only $74 \%$ of all lumens; however, the authors concluded that the differential time to positivity was the most simple technique to perform. As a result of the high specificity of the method, they recommended its use as a first-line approach, with the endoluminal brush technique reserved for cases in which blood cannot be obtained from the device.

In a prospective cohort study of 204 CVCs suspected of causing catheter-related bloodstream infections in critically ill patients, Bouza et al. $\left[37^{\bullet \bullet}\right]$ compared the performance of three methods that allowed the device to remain in situ. The sensitivity and specificity of cultures of swabs from the insertion site and from the hub were 78.6 and $92.0 \%$, respectively; for differential quantitative blood cultures, 71.4 and $97.7 \%$, respectively; and for the differential time to positivity, 96.4 and $90.3 \%$, respectively. From these data, the authors argued that convenience in different medical contexts, the use of resources, and expertise should determine the choice of a technique. As a result of the ease of performance, low cost, and wide availability, they recommended combining semiquantitative superficial cultures and peripheral vein blood cultures for the screening of devices suspected of causing infection, and to use differential quantitative blood cultures as a confirmatory method.

Those studies suggested that the choice of a precise microbiological method, or of the eventual combinations of some of them, should be made according to technical availability and should be integrated in strategies discussed between clinicians and microbiologists in order to provide useful information at the bedside. In addition, economic considerations, such as cost-effectiveness, may also be taken into account.

\section{Recommendations of experts}

Experts have proposed algorithms taking into account most of these difficulties to help clinicians in the diagnosis of intravascular access-related infections. Worthington and Eliott [4] suggested obtaining for every patient two sets of paired blood cultures drawn through 


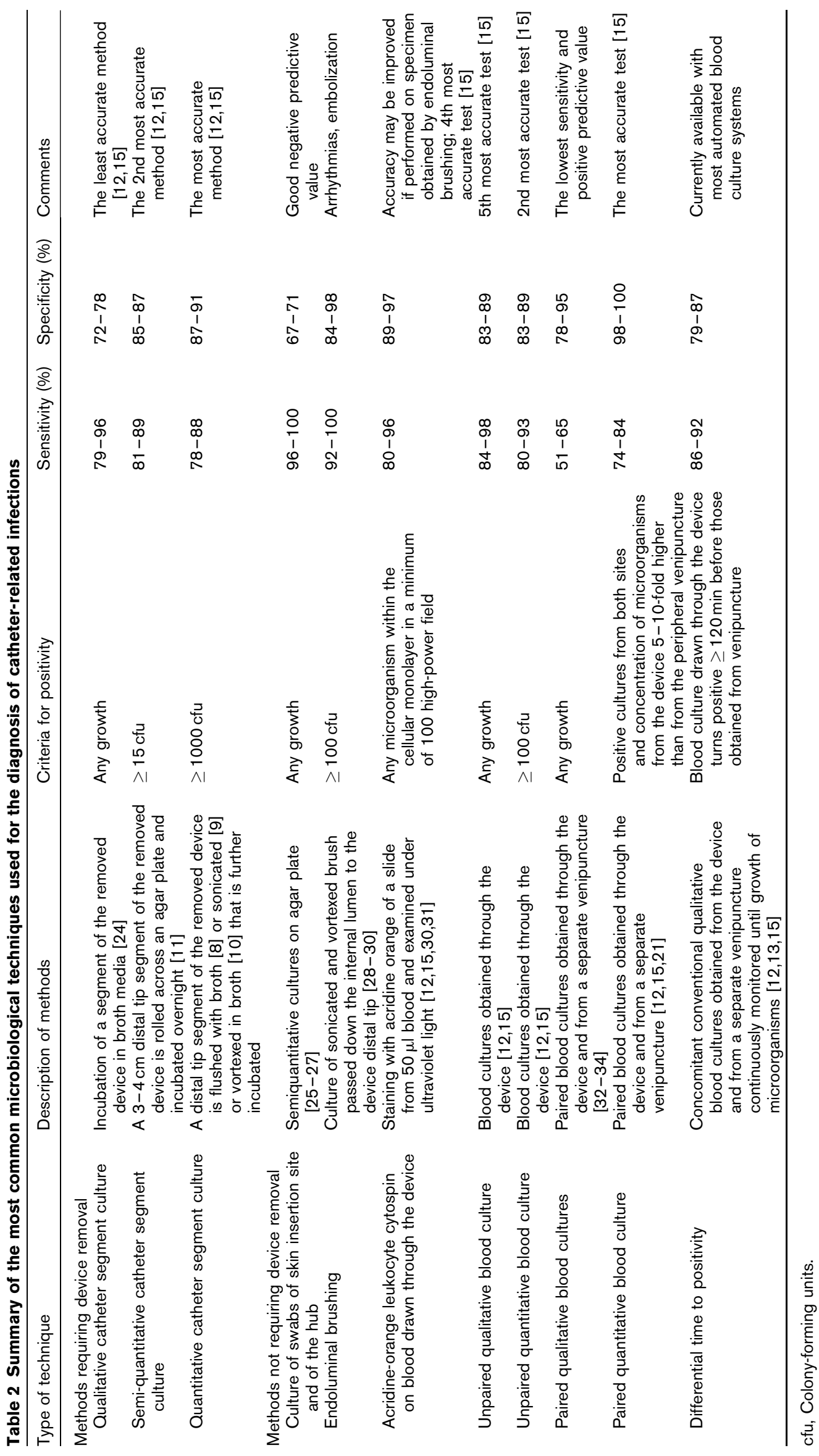


the device and peripherally from venipuncture. A sufficient volume of blood collected per set $(20-30 \mathrm{ml})$ and inoculated into both aerobic and anaerobic media should allow the identification of $99 \%$ of detectable bacteraemias. In cases in which clinical judgement mandates the removal of the device, quantitative cultures should provide information likely to confirm the diagnosis. If the intravascular access is not removed, the differential time to positivity is then recommended as the first-line method, followed by quantitative blood cultures. Alternatively, if only qualitative blood cultures are available, the authors strongly recommend performing additional tests, such as culture of the device, to improve the sensitivity of the method. In any cases of positive microbiological cultures, the authors recommend applying more strict criteria in the presence of coagulase-negative staphylococci likely to reflect only contamination [4].

The International Sepsis Forum Consensus Conference on Definitions of Infection in the Intensive Care Unit suggested taking into account risk factors likely to increase the probability of an infection being related to an intravascular access in its management. Removal of the device is strongly recommended in the presence of severe sepsis or septic shock with episodes of hypotension when the catheter is flushed, with the catheter in place for more than 7 days or inserted in non-sterile conditions, or with evidence of exit-site infection. In the absence of bacteraemia but positive culture of the tip of the device, the hub or the exit-site, the infection is scored as a possible clinical catheter-related sepsis. It is scored as a catheter-related sepsis with bacteriological confirmation in the presence of bacteraemia with common skin commensals and positive culture of the tip or exit site with the same microorganism [18].

\section{Conclusion}

The distinction between device-associated and devicerelated infections has improved our ability to diagnose clinical infections at the bedside, and has clarified the situations in which further microbiological diagnostic methods should be performed. Despite the usefulness of the recently proposed algorithms, however, they all include some simplifications, and none has been validated in prospective clinical trials. In addition, the potential impact of concomitant systemic antibiotic treatment or the use of antiseptic/antimicrobial-coated devices on the accuracy of microbiological techniques remains to be determined.

Accordingly, precise diagnostic criteria should be clearly discussed and defined in each institution by a close collaboration between clinicians and microbiologists. They should then be used for eventual benchmarking and further integrated into global strategies targeted at the prevention of vascular access-related or associated infections.

\section{References and recommended reading}

Papers of particular interest, published within the annual period of review, have been highlighted as:

- of special interest

-• of outstanding interest

Additional references related to this topic can also be found in the Current

World Literature section in this issue (p. 436).

1 National Nosocomial Infections Surveillance (NNIS). System report, data summary from January 1992 through June 2004, issued October 2004 Am J Infect Control 2004; 32: 470-485.

2 Warren DK, Quadir WW, Hollenbeak CS, et al. Attributable cost of catheter- associated bloodstream infections among intensive care patients in a nonteaching hospital. Crit Care Med 2006; 34:2084-2089.

After adjusting for potential confounders in a cohort of 41 catheter-associated bloodstream infections, these infections resulted in an attributable cost of US\$11971, ICU length of stay of 2.41 days, and hospital length of stay of 7.54 days.

3 Wenzel RP, Edmond MB. Team-based prevention of catheter-related infec- $\quad$ tions. N Engl J Med 2006; 355:2781-2783.

In a well-documented editorial, after new estimations of the epidemiology of catheter-related infection in the United States, the authors recommend to the medical community to adhere to such concepts as caring behaviour that consistently lead to safety and comfort for patients.

4 Worthington T, Elliott TS. Diagnosis of central venous catheter related infection in adult patients. J Infect 2005; 51:267-280.

5 Linares J. Diagnosis of catheter-related bloodstream infection: conservative

- techniques. Clin Infect Dis 2007; 44:827-829.

An editorial about the difficulties in diagnosing catheter-related infections.

6 O'Grady NP, Alexander M, Dellinger EP, et al. Guidelines for the prevention of intravascular catheter-related infections. Centers for Disease Control and Prevention. Morb Mortal Wkly Rep 2002; 51:1-29.

7 Hugonnet S, Sax H, Eggimann P, et al. Clinical sepsis: epidemiology of an unrecognized entity. Emerg Infect Dis 2004; 10:76-81.

8 Cleri DJ, Corrado ML, Seligman SJ. Quantitative culture of intravenous catheters and other intravascular inserts. J Infect Dis 1980; 141:781-786.

9 Sherertz RJ, Raad II, Belani A, et al. Three-year experience with sonicated vascular catheter cultures in a clinical microbiology laboratory. J Clin Microbiol 1990; 28:76-82.

10 Brun-Buisson C, Abrouk F, Legrand $\mathrm{P}$, et al. Diagnosis of central venous catheter-related sepsis. Arch Intern Med 1987; 147:873-877.

11 Maki DG, Weise CE, Sarafin HW. A semiquantitative culture method for identifying intravenous-catheter-related infection. N Engl J Med 1977; 296 : 1305-1309.

12 Siegman-Igra Y, Anglim AM, Shapiro DE, et al. Diagnosis of vascular catheterrelated bloodstream infection: a meta-analysis. J Clin Microbiol 1997; 35: 928-936.

13 Blot F, Nitenberg G, Chachaty E, et al. Diagnosis of catheter-related bacteremia: a prospective comparison of the time to positivity of hub-blood versus peripheral-blood cultures. Lancet 1999; 354:1071-1077.

14 Garner JS, Jarvis WR, Emori TG, et al. CDC definitions for nosocomial infections. Am J Infect Control 1988; 16:128-140.

15 Safdar N, Fine JP, Maki DG. Meta-analysis: methods for diagnosing intravascular device-related bloodstream infection. Ann Intern Med 2005; 142:451-466.

16 Maki DG, Kluger DM, Crnich CJ. The risk of bloodstream infection in adults -. with different intravascular devices: a systematic review of 200 published prospective studies. Mayo Clin Proc 2006; 81:1159-1171.

A comprehensive determination of the absolute (expressed as episodes per 100 devices) and relative risks (expressed as episodes per 1000 device-days) of microbiologically documented bloodstream infections associated with the use of various types of intravascular device, extracted and pooled from a systematic review of 200 published studies over four decades.

17 Bone RC, Balk RA, Cerra FB, et al. Definitions for sepsis and organ failure and guidelines for the use of innovative therapies in sepsis. the ACCP/SCCM consensus conference committee. American College of Chest Physicians/ Society of Critical Care Medicine. Chest 1992; 101:1644-1655.

18 Calandra T, Cohen J. The international sepsis forum consensus conference on definitions of infection in the intensive care unit. Crit Care Med 2005; 33: 1538-1548. 
19 Cook D, Randolph A, Kernerman P, et al. Central venous catheter replacement strategies: a systematic review of the literature. Crit Care Med 1997; 25:1417-1424.

20 Timsit JF. Scheduled replacement of central venous catheters is not necessary. Infect Control Hosp Epidemiol 2000; 21:371-374.

21 Mermel LA, Farr BM, Sherertz RJ, et al. Guidelines for the management of intravascular catheter-related infections. Clin Infect Dis 2001; 32:12491272.

22 Brun-Buisson C. Suspected central venous catheter-associated infection: can the catheter be safely retained? Intensive Care Med 2004; 30:10051007.

23 Rijnders BJ, Peetermans WE, Verwaest C, et al. Watchful waiting versus immediate catheter removal in ICU patients with suspected catheter-related infection: a randomized trial. Intensive Care Med 2004; 30:1073-1080.

24 Druskin MS, Siegel PD. Bacterial contamination of indwelling intravenous polyethylene catheters. JAMA 1963; 185:966-968; 966-968.

25 Cercenado E, Ena J, Rodriguez-Creixems M, et al. A conservative procedure for the diagnosis of catheter-related infections. Arch Intern Med 1990; 150:1417-1420.

26 Atela I, Coll P, Rello J, et al. Serial surveillance cultures of skin and catheter hub specimens from critically ill patients with central venous catheters: molecular epidemiology of infection and implications for clinical management and research. J Clin Microbiol 1997; 35:1784-1790.

27 Bouza E, Munoz P, Burillo A, et al. The challenge of anticipating catheter tip colonization in major heart surgery patients in the intensive care unit: are surface cultures useful? Crit Care Med 2005; 33:1953-1960.

28 Kite $\mathrm{P}$, Dobbins $\mathrm{BM}$, Wilcox $\mathrm{MH}$, et al. Evaluation of a novel endoluminal brush method for in situ diagnosis of catheter related sepsis. J Clin Pathol 1997; 50:278-282.
29 Tighe MJ, Kite P, Fawley WN, et al. An endoluminal brush to detect the infected central venous catheter in situ: a pilot study. BMJ 1996; 313:1528-1529.

30 Kite P, Dobbins BM, Wilcox MH, McMahon MJ. Rapid diagnosis of centralvenous-catheter-related bloodstream infection without catheter removal. Lancet 1999; 354:1504-1507.

31 Rushforth JA, Hoy CM, Kite P, Puntis JW. Rapid diagnosis of central venous catheter sepsis. Lancet 1993; 342:402-403.

32 Martinez JA, DesJardin JA, Aronoff M, et al. Clinical utility of blood cultures drawn from central venous or arterial catheters in critically ill surgical patients. Crit Care Med 2002; 30:7-13.

33 Beutz M, Sherman G, Mayfield J, et al. Clinical utility of blood cultures drawn from central vein catheters and peripheral venipuncture in critically ill medical patients. Chest 2003; 123:854-861.

34 Tanguy $M$, Seguin $P$, Laviolle $B$, et al. Hub qualitative blood culture is useful for diagnosis of catheter-related infections in critically ill patients. Intensive Care Med 2005; 31:645-648.

35 Marik PE. Fever in the ICU. Chest 2000; 117:855-869.

36 Catton JA, Dobbins BM, Kite $P$, et al. In situ diagnosis of intravascular catheter-related bloodstream infection: a comparison of quantitative culture, differential time to positivity, and endoluminal brushing. Crit Care Med 2005; 33:787-791.

37 Bouza E, Alvarado N, Alcala L, et al. A randomized and prospective study of 3

- procedures for the diagnosis of catheter-related bloodstream infection without catheter withdrawal. Clin Infect Dis 2007; 44:820-826.

A prospective comparison of three microbiological methods avoiding the removal of the intravascular device in 204 suspicions of CVC-related infection. The authors recommended combining semiquantitative superficial cultures and peripheral vein blood cultures to screen for catheter-related bloodstream infections, leaving differential quantitative blood cultures as a confirmatory and more specific technique. 